\title{
A Gold@Polydopamine Core-Shell Nanoprobe for Long-Term Intracellular Detection of MicroRNAs in Differentiating Stem
}

\section{Cells}

Chun Kit K. Choi, Jinming Li, ${ }^{\dagger} \|$ Kongchang Wei, $" \|$ Yang J. Xu, Lok Wai C. Ho, Meiling Zhu, ${ }^{\dagger}$ Kenneth K. W. To, ${ }^{\S}$ Chung Hang J. Choi ${ }^{*, *, \|}$ and Liming Bian ${ }^{*,+, \|}$

Department of Mechanical and Automation Engineering (Biomedical Engineering), Department of Electronic Engineering (Biomedical Engineering), School of Pharmacy, "Shun Hing Institute of Advanced Engineering, The Chinese University of Hong Kong, Shatin, New Territories, Hong Kong, China

*To whom correspondence should be addressed. Email: jchchoi@ee.cuhk.edu.hk or lbian@mae.cuhk.edu.hk. 


\section{EXPERIMENTAL SECTION}

\section{Analysis of Osteogenic Marker Gene Expression}

For gene expression analysis, hMSCs were grown in a 6-well plate (SPL Lifescience). Upon reaching confluence, cells were harvested and homogenized in Trizol Reagent (Invitrogen) with a tissue grinder. Total RNA in cells was extracted according to the manufacturer's instructions, and the RNA concentration was determined by a UV spectrometer (Nanodrop Technologies). $500 \mathrm{ng}$ of the extracted RNA was reverse-transcribed into cDNA with the Revert Aid First Strand cDNA Synthesis Kit (Thermo Scientific) for each sample. Real-time polymerase chain reaction (RT-PCR) was performed using Taqman primers and probes specific for RUNX2 and ALP (early marker genes for osteogenesis) and GAPDH (a housekeeping gene) with an Applied Biosystems Step One Plus real-time PCR system. The relative gene expression of each marker gene was calculated by the quantitation-comparative CT $\left(\Delta \Delta_{\mathrm{CT}}\right)$ method. Fold difference between different groups was calculated using the expression $2^{-\Delta \Delta \mathrm{Ct}}$. Each sample expression was internally normalized to the GAPDH data and each group expression was normalized to the expression level of control group of the same culture time. Sequence information of the primer and probe of the marker $A L P$ used in the analysis are listed in Table $\mathrm{S} 4$. Note that the respective sequences for the RUNX2 marker are proprietary and not disclosed by the company (Applied Biosystem).

\section{Immunofluorescence Staining of RUNX2 and ALP Staining}

hMSCs were grown in a coverglass bottom dish and allowed to differentiate in osteogenic induction medium. At each selected time point (i.e., $1 \mathrm{~d}, 3 \mathrm{~d}, 5 \mathrm{~d}$, and $7 \mathrm{~d}$ ), cells were fixed with $4 \%$ formalin for $15 \mathrm{~min}$ at room temperature, rinsed with DPBS (Invitrogen) for three times, permeabilized with 2\% triton-X 100 (Sigma Aldrich) in DPBS for $10 \mathrm{~min}$ at room temperature, and then blocked with $1 \%$ bovine serum albumin (BSA) (Sigma Aldrich) at $37{ }^{\circ} \mathrm{C}$ for $1 \mathrm{~h}$. To visualize the change in the nuclear RUNX2 level, cells were immuno-stained with 1:100 primary antibodies (Santa Cruz Biotechnology, sc-101145) against RUNX2 in $0.5 \%$ BSA in PBS for overnight at $4{ }^{\circ} \mathrm{C}$. Following that, secondary antibody labeling was performed by incubation in 1:200 labeled goat anti-mouse or anti-rabbit IgG (Life Technologies, A-11001) containing Alexa488-phalloidin in 0.5\% BSA in PBS for $2 \mathrm{~h}$ at room temperature. The stained cells were thoroughly rinsed with DPBS and imaged under a Nikon Eclipse TI microscope (Nikon).

To stain ALP, cells were incubated in the staining solution containing $4 \%$ of naphthol AS-MX phosphate (Sigma Aldrich) in Fast Blue RR Salt solution (Sigma Aldrich) in deionized water for $20 \mathrm{~min}$ at room temperature in dark. Next, hMSCs were rinsed in PBS and imaged under microscopy with constant illumination. 


\section{Quantification of Fluorescently-labeled Oligonucleotides Loading}

The concentration of the AuNP stock solution was first measured by using a Cary 5000 UV-vis spectrophotometer (Agilent). Triplicate measurements were performed to obtain the data. These absorbance values were then related to the nanoparticle concentration via Beer's Law $(A=\varepsilon b c)$. The wavelength of the absorbance maximum $\left(\lambda_{\max }\right)$ was determined as $530 \mathrm{~nm}$ and the respective extinction coefficient $(\varepsilon)$ used for the calculation was extracted from previous report. ${ }^{1}$ To determine the concentration of immobilized fluorescently-labeled oligonucleotides, fluorescence measurement was performed as depicted in Scheme S1. Triplicate measurements were performed to obtain the data. The number of the oligonucleotide strands per particle is calculated by dividing the concentration of loaded fluorescently-labeled hpDNAs by the concentration of nanoparticles.

\section{Inductively Coupled Plasma Optical Emission Spectrometry}

To quantify the amount of nanoparticles (based on the Au content) inside the treated hMSCs after initial cellular entry, cells were grown in a 24-well plate (SPL Lifescience) to $80-90 \%$ confluence and incubated with $\sim 0.025 \mathrm{nM}$ of either Au@PDA NPs or Au@PDA-hpDNA NPs for 24 h. At each selected time point (i.e., 1 d, 3 d, and 5 d), cells were washed thoroughly with PBS. Following that, cell pellets were obtained and then digested in $0.25 \mathrm{~mL}$ of freshly prepared aqua regia $\left(\mathrm{HCl}: \mathrm{HNO}_{3}=1: 3, \mathrm{v} / \mathrm{v}\right.$ ) at $55^{\circ} \mathrm{C}$ for $30 \mathrm{~min}$. After adding $0.08 \mathrm{~mL}$ of $1000 \mathrm{ppm}$ indium (internal standard; AccuStandard) and $3.52 \mathrm{~mL}$ of matrix solution $(2 \% \mathrm{HCl}$ and $2 \%$ $\mathrm{HNO}_{3}$ ), the atomic Au content in the resultant solution was determined by an Optima 4300DV inductively coupled plasma optical emission spectrometer (PerkinElmer). The data obtained from untreated hMSCs were used for background correction. 


\section{Table S1}

\begin{tabular}{|l|l|}
\hline Name & \multicolumn{1}{|l|}{ Sequence $\left(\mathbf{5}^{\prime} \rightarrow \mathbf{3}^{\prime}\right)$} \\
\hline hsa-miR-29b & UAG CAC CAU UUG AAA UCA GUG UU \\
hsa-miR-31 & AGG CAA GAU GCU GGC AUA GCU \\
\hline $\begin{array}{l}\text { DNA analog of } \\
\text { hsa-miR-29b }\end{array}$ & TAG CAC CAT TTG AAA TCA GTG TT \\
\hline $\begin{array}{l}\text { FITC-labeled } \\
\text { hpDNA-29b }\end{array}$ & $\begin{array}{l}\text { FITC-CCG GGT AAC ACT GAT TTC AAA TGG TGC TA } \\
\text { ACCG }\end{array}$ \\
\hline $\begin{array}{l}\text { Cy3-labeled } \\
\text { hpDNA-31 }\end{array}$ & \begin{tabular}{l} 
Cy3-CCG GGT AGC TAT GCC AGC ATC TTG CCT ACC \\
\hline FITC-labeled \\
scrambled DNA
\end{tabular} \\
\hline $\begin{array}{l}\text { FITC-GCT GCT TTA TAG AGC CTG TTG A } \\
\text { CAG TGG TTT TAC CCT ATG GTA G }\end{array}$ \\
\hline
\end{tabular}

Table S1. DNA sequences employed for the extra- and intracellular detection of miRNAs. All nanoprobes are prepared by immobilizing the hpDNA strands onto the surface of Au@PDA NPs upon $1 \mathrm{~h}$ of incubation. The scrambled DNA sequence refers to a DNA sequence with the following properties: (i) bears the same nucleotide composition as the probe sequence, (ii) has no complex sequence, and (iii) shows the weakest (or no) matches with any mRNA in the mRNA pool for human. Base pairs in the stem region of the hpDNA probes are underlined for clarity. $\mathrm{Cy} 3=$ cyanine 3 , FITC $=$ fluorescein isothiocyanate. 


\section{Table S2}

\begin{tabular}{l|l|l|l|} 
Sample & $\begin{array}{l}\text { Hydrodynamic size } \\
{[\mathbf{n m}] \text { (PDI) }}\end{array}$ & $\begin{array}{l}\text { Zeta potential } \\
{[\mathrm{mV}]}\end{array}$ & $\begin{array}{l}\text { Number of } \\
\text { oligonucleotides } \\
\text { per particle }\end{array}$ \\
\hline $\begin{array}{l}\text { Au@PDA NPs } \\
\text { (Before the DNA } \\
\text { immobilization) }\end{array}$ & $56.1 \pm 1.6(0.19)$ & $-39.0 \pm 1.5$ & N. A. ${ }^{\text {a }}$ \\
\hline $\begin{array}{l}\text { Au@PDA-hpDNA } \\
\text { NPs (After the DNA } \\
\text { immobilization) }\end{array}$ & $68.5 \pm 1.2(0.14)$ & $-28.3 \pm 2.3$ & $256 \pm 16$ \\
\hline
\end{tabular}

Table S2. Physiochemical properties of Au@PDA-hpDNA NPs. For comparison, characterization data of the bare Au@PDA NPs are shown in the upper row. Results are given in mean \pm SD of 10 measurements. $\mathrm{PDI}=$ poly-dispersity index. ${ }^{\mathrm{a}}$ Measurement is not applicable to the sample. 
Table S3

\begin{tabular}{|l|l|l|l|l|}
\hline Methods & $\begin{array}{l}\text { Minimal Time } \\
\text { for } \\
\text { Distinguishing } \\
\text { the } \\
\text { Differentiation } \\
\text { Status of } \\
\text { hMSCs }\end{array}$ & $\begin{array}{l}\text { Is Cell Lysis } \\
\text { Required? }\end{array}$ & $\begin{array}{l}\text { Live Cell } \\
\text { Monitoring? }\end{array}$ & $\begin{array}{l}\text { Long-term } \\
\text { Monitoring? }\end{array}$ \\
\hline qRT-PCR of Genes & $3 \mathrm{~d}$ & Yes & No & No \\
\hline $\begin{array}{l}\text { Staining of } \\
\text { Proteins }\end{array}$ & $5-7 \mathrm{~d}$ & No & No & No \\
\hline $\begin{array}{l}\text { Commercial RNA } \\
\text { Detection Probe } \\
\text { e.g., SmartFlare }\end{array}$ & $1 \mathrm{~d}$ & No & Yes & $\begin{array}{l}\text { Up to 3 dif } \\
\text { single } \\
\text { administration } \\
\text { is applied to } \\
\text { cells }\end{array}$ \\
\hline $\begin{array}{l}\text { Au@PDA-hpDNA } \\
\text { Nanoprobes }\end{array}$ & $1 \mathrm{~d}$ & No & Yes & $\begin{array}{l}\text { Up to 5 dif } \\
\text { single } \\
\text { administration } \\
\text { is applied to } \\
\text { cells }\end{array}$ \\
\hline
\end{tabular}

Table S3. Summary of the performance of conventional methods and nanoparticle-based methods used for the monitoring of the differentiation status of hMSCs. 


\section{Table S4}

\begin{tabular}{|l|l|l|l|}
\multicolumn{1}{c}{ Gene } & \multicolumn{1}{l}{ Forward Primer } & \multicolumn{1}{l|}{ Reverse Primer } \\
$A L P$ & $\begin{array}{l}\text { CGGAACTCCTGAC } \\
\text { CCTTGAC }\end{array}$ & $\begin{array}{l}\text { TGTTCAGCTCGTACTG } \\
\text { CATGTC }\end{array}$ & $\begin{array}{l}\text { TCGAAGAGACCCA } \\
\text { ATAGGT }\end{array}$ \\
\hline
\end{tabular}

Table S4. Sequence information of the primers and probe of osteogenic marker gene employed in the RT-PCR analysis. $A L P=$ alkaline phosphatase. 


\section{Figure S1}
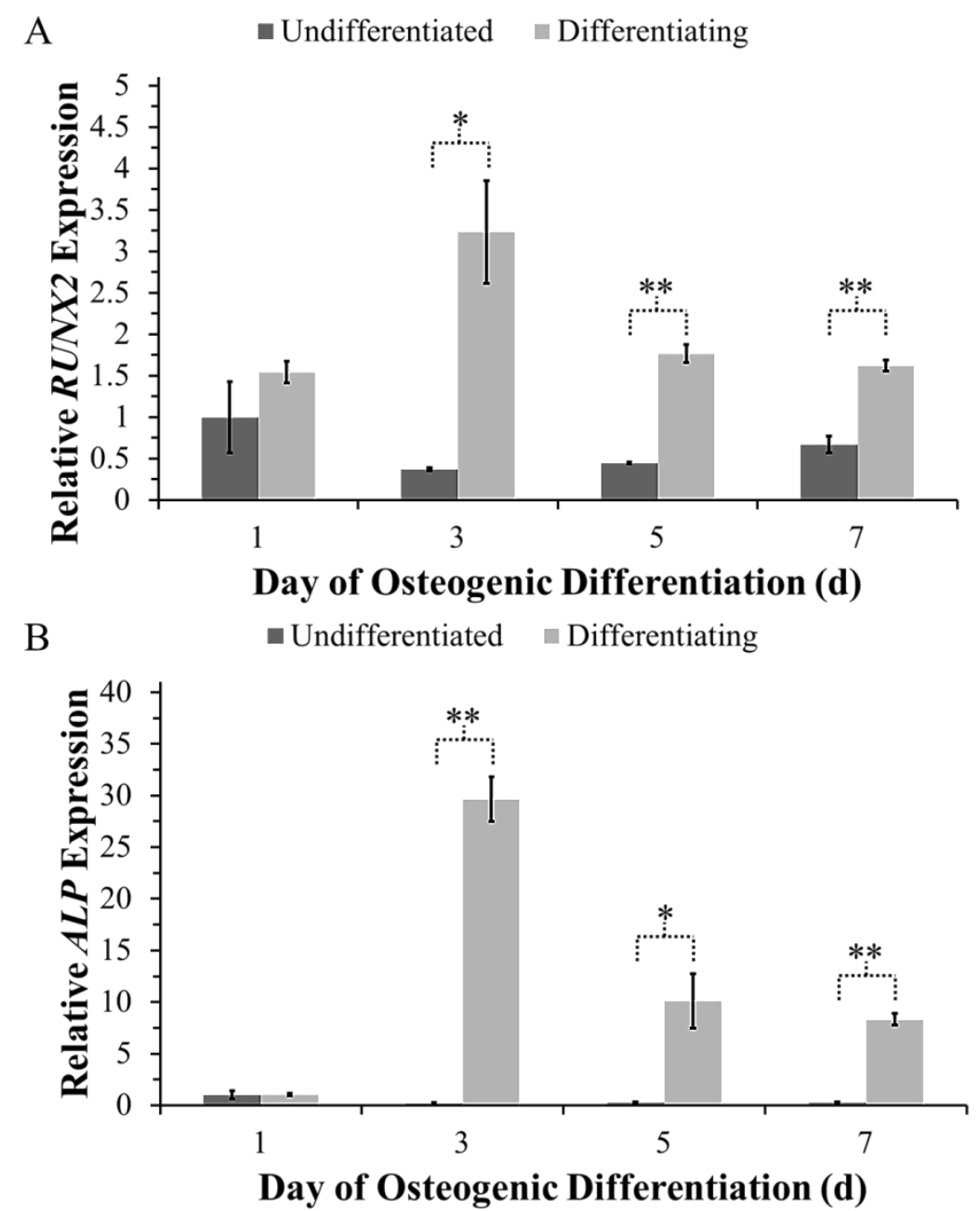

Figure S1. qRT-PCR measurements of two early osteogenic marker genes, namely (A) RUNX2 and (B) ALP, in hMSCs. Our results show that both the RUNX2 and ALP show differential gene expression between the differentiating and undifferentiated hMSCs upon $3 \mathrm{~d}$ of osteogenic induction. qRT-PCR is performed using an off-target gene $G A P D H$ as the internal control. Data obtained from three independent measurements are presented as mean $\pm \mathrm{SD} .{ }^{*}=\mathrm{p}<0.05 ; * *=\mathrm{p}<0.005$. RUNX2 $=$ Runt-related transcription factor 2. $A L P=$ alkaline phosphatase. $G A P D H=$ glyceraldehyde 3-phosphate dehydrogenase. 


\section{Figure S2}

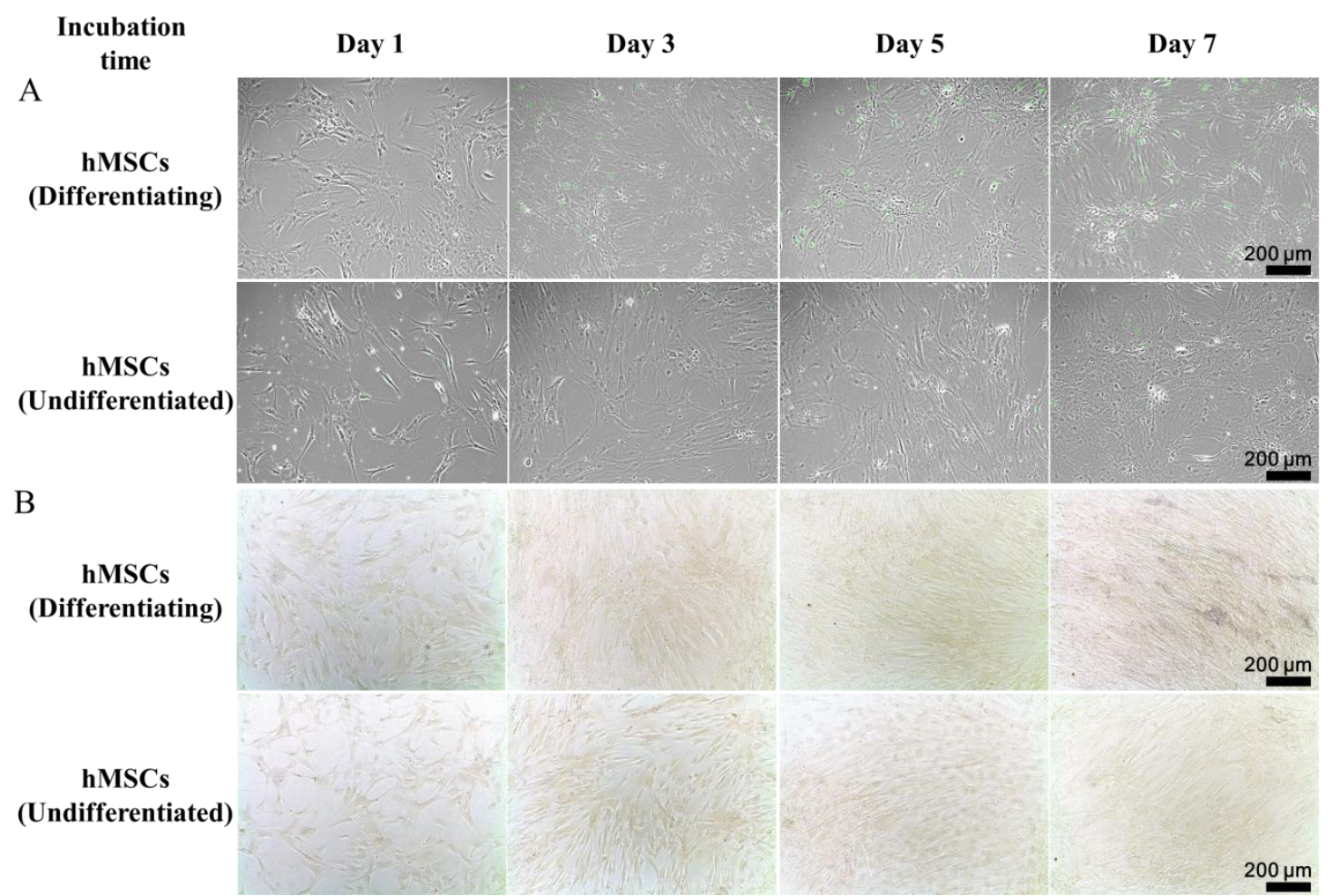

Figure S2. Conventional methods of monitoring the differentiation progress of hMSCs. (A) Results of immunofluorescence staining against RUNX2. Significant difference of the nuclear fluorescence (green) between the differentiating and undifferentiated hMSCs is observed upon $5 \mathrm{~d}$ of osteogenic induction. (B) Results of histochemical staining of ALP. Significant difference between the differentiating and undifferentiated hMSCs is observed upon $7 \mathrm{~d}$ of osteogenic induction. Positively stained cells appear as purple in color. Scale bar is $200 \mu \mathrm{m}$. Note that one cannot observe major differences between the differentiating and undifferentiated hMSCs in the early stage of osteogenesis by using these two conventional methods. 


\section{Figure S3}
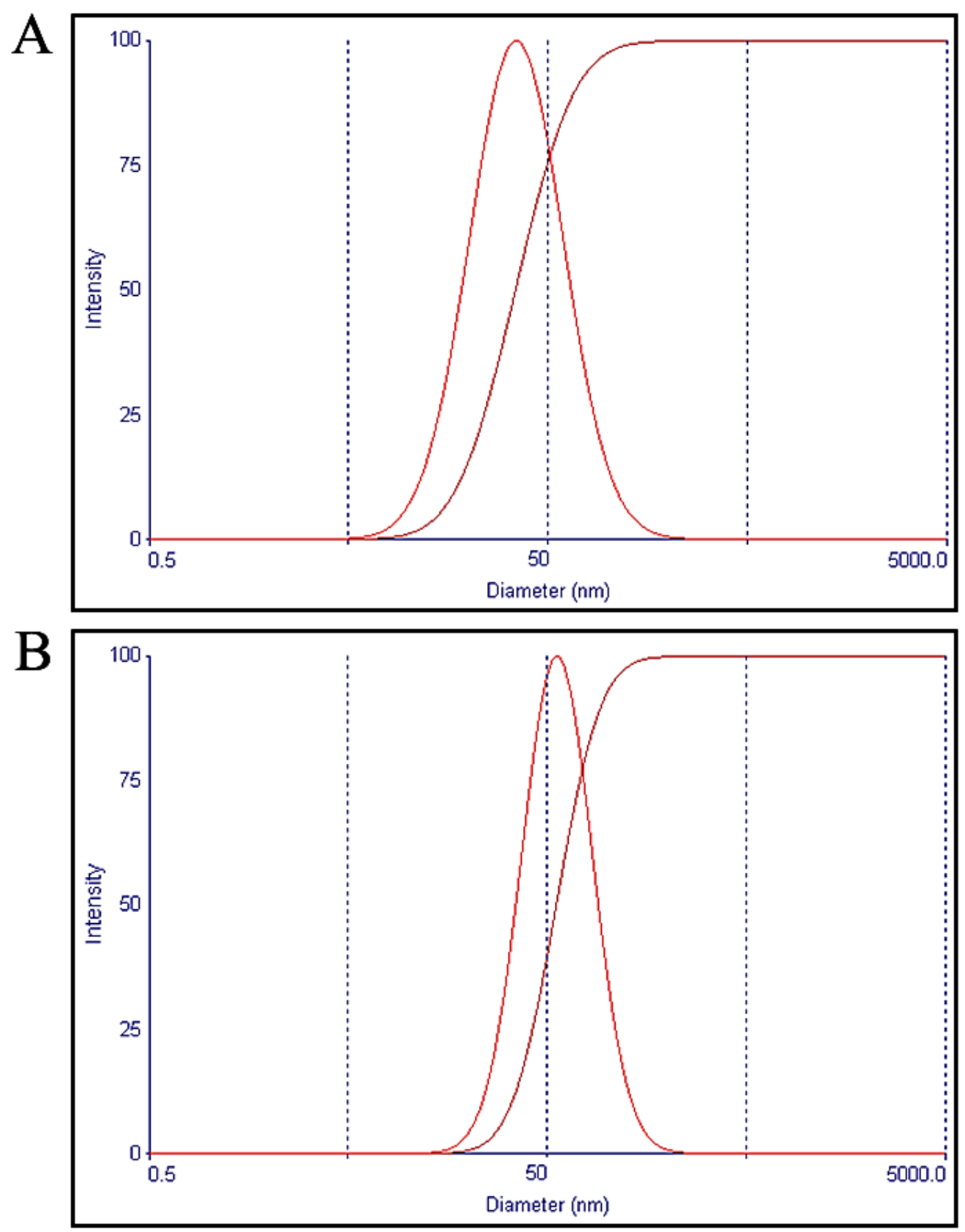

Figure S3. Dynamic light scattering (DLS) measurements of (A) AuNPs and (B) Au@PDA NPs in water at $25{ }^{\circ} \mathrm{C}$. The hydrodynamic size of AuNPs increases by around $12 \mathrm{~nm}$ after coating with the thin PDA shell, which is consistent with the TEM measurements (see Figure 1B). 


\section{Figure S4}

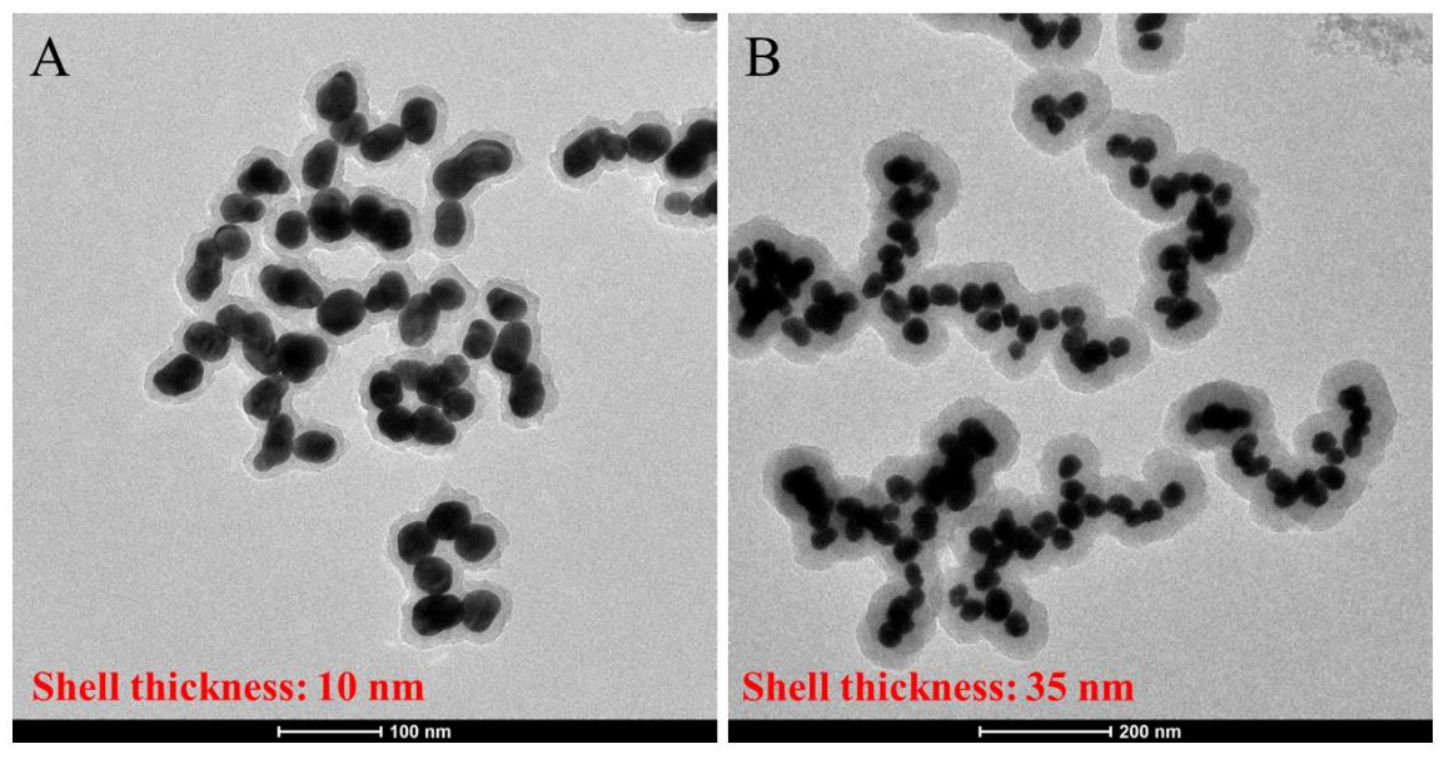

Figure S4. Representative TEM images of Au@PDA NPs with different PDA shell thicknesses. (A) AuNPs are coated with a PDA shell of $\sim 10 \mathrm{~nm}$ thickness in 0.1 $\mathrm{mg} / \mathrm{mL}$ of dopamine solution. (B) AuNPs are coated with a PDA shell of $\sim 35 \mathrm{~nm}$ thickness in $0.4 \mathrm{mg} / \mathrm{mL}$ of dopamine solution. The PDA shell thickness can be tuned by simply varying the dopamine concentration while keeping the reaction time constant at $1 \mathrm{~h}$. 


\section{Figure S5}

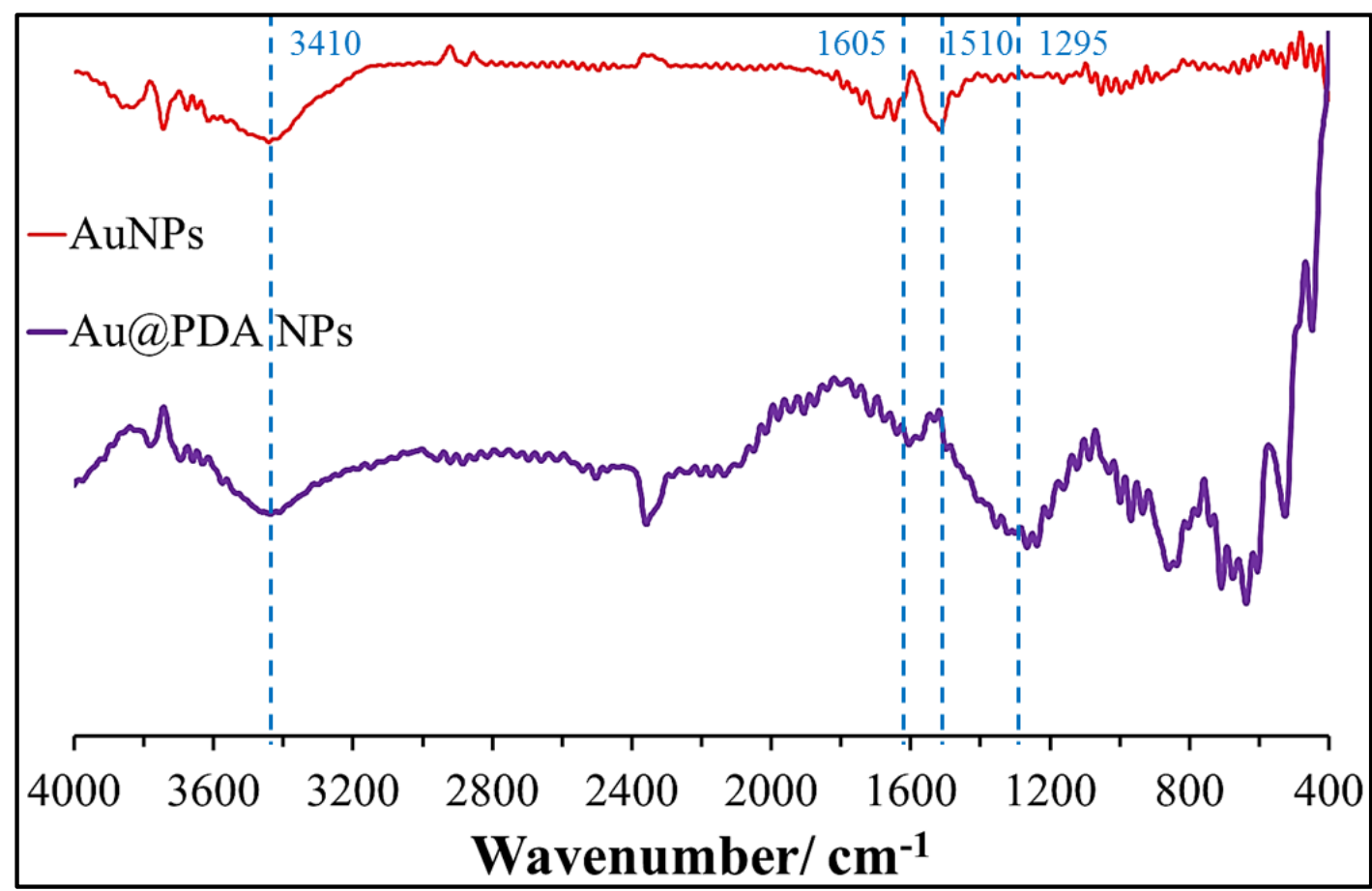

Figure S5. FT-IR spectra of AuNPs (red) and Au@PDA NPs (purple). Newly emerged absorption bands at $3410 \mathrm{~cm}^{-1}$ (stretching vibration of phenolic $\mathrm{O}-\mathrm{H}$ and $\mathrm{N}-$ $\mathrm{H}$ ), $1605 \mathrm{~cm}^{-1}$ (stretching vibration of aromatic ring and bending vibration of $\mathrm{N}-\mathrm{H}$ ), $1510 \mathrm{~cm}^{-1}$ (shearing vibration of $\mathrm{N}-\mathrm{H}$ ), and $1295 \mathrm{~cm}^{-1}$ (stretching vibration of phenolic $\mathrm{C}-\mathrm{O}$ ) are observed after coating AuNPs with PDA, indicating the presence of the PDA shell on the surface of AuNPs. 


\section{Figure S6}

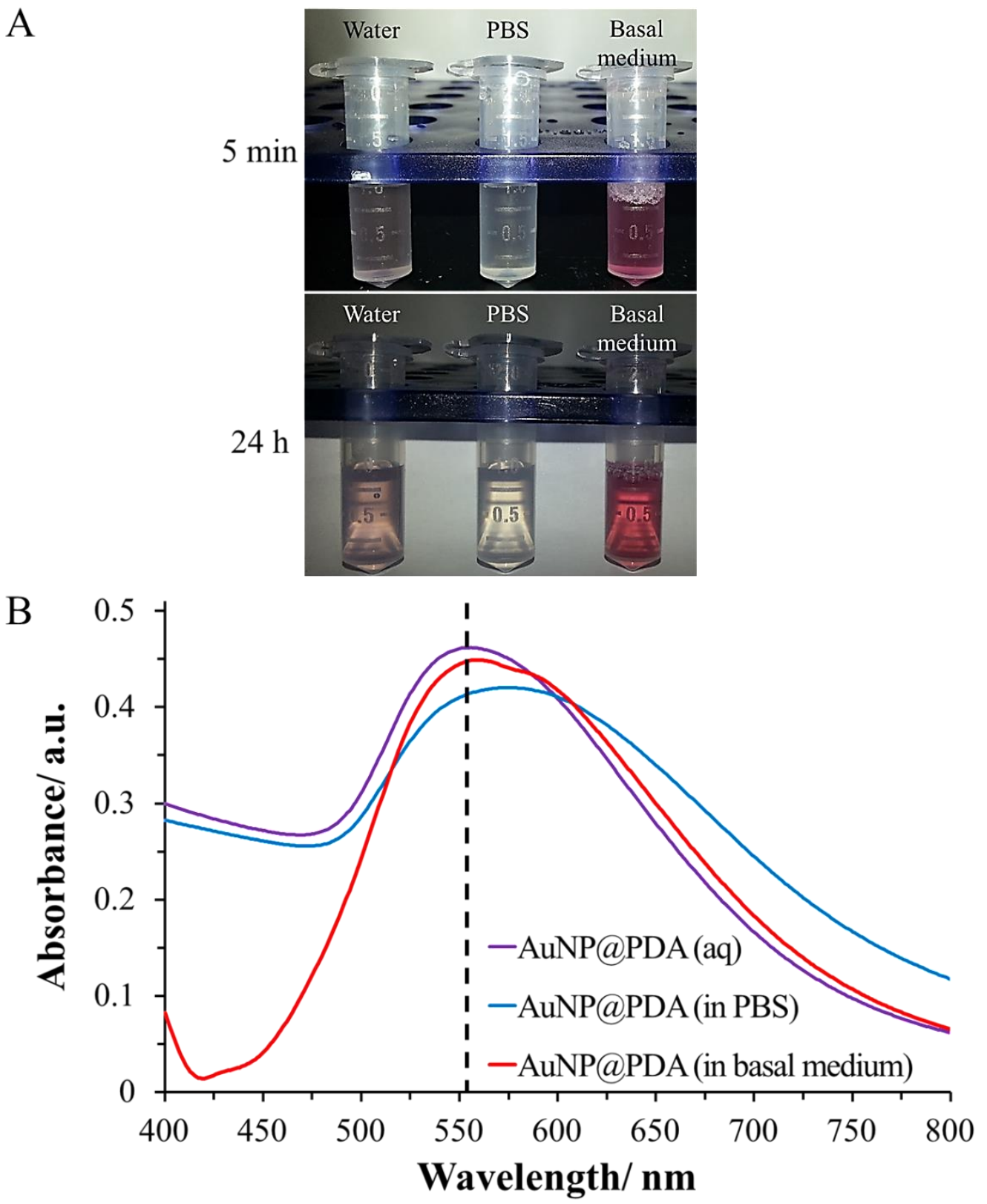

Figure S6. Stability test of Au@PDA NPs in water, PBS, and basal medium ( $\alpha$-MEM $+10 \%$ FBS). (A) Photographs of Au@PDA NPs before and after incubation in different solutions for $24 \mathrm{~h}$. The solutions remain clear without obvious aggregation. $\alpha-\mathrm{MEM}=$ alpha minimum essential medium, FBS $=$ fetal bovine serum. (B) UV-vis spectra of the solutions shown in (A). No significant red-shift of the $\lambda_{\max }$ is observed in all the solutions with respect to the peak of the stock prepared in water (indicated by the black line), supporting our observation that no obvious aggregation happens. 


\section{Figure S7}

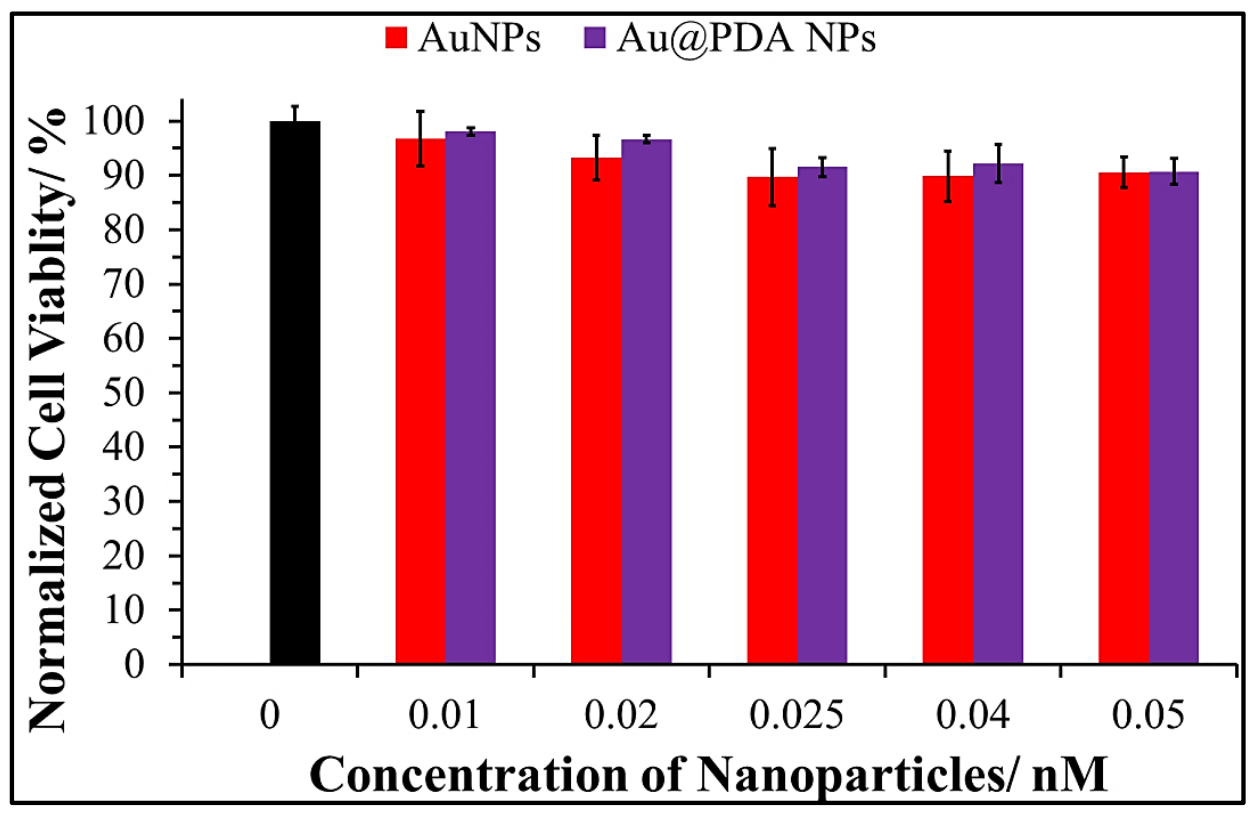

Figure S7. Cytotoxicity of hMSCs evaluated by the Alamar blue assay after incubation with AuNPs or Au@PDA NPs at different concentrations (expressed as Au content obtained from ICP-OES measurement) for $24 \mathrm{~h}$. Results are normalized by using the cytotoxicity data of untreated hMSCs. Data obtained from six independent measurements are presented as mean \pm SD. 


\section{Figure S8}

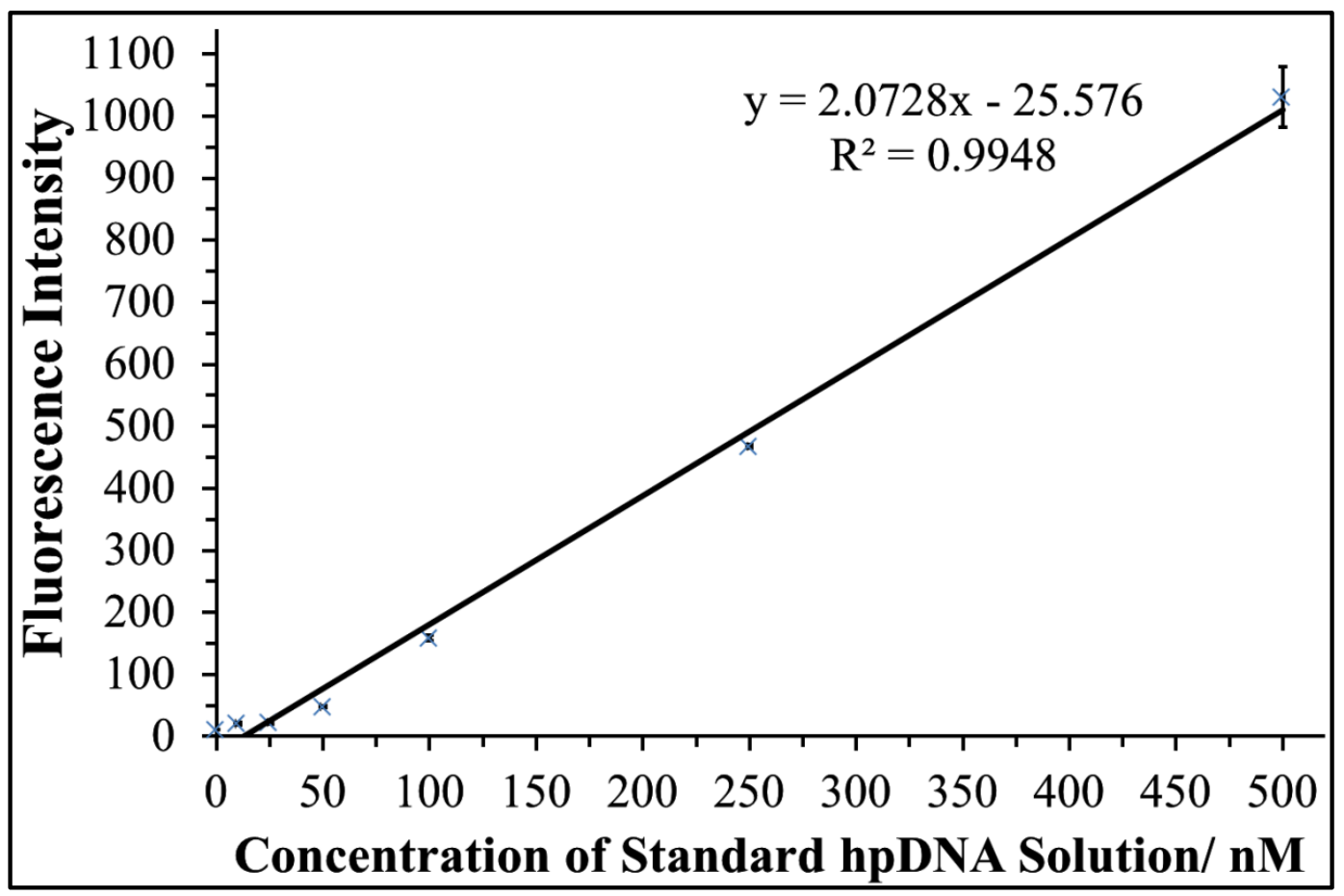

Figure S8. Standard linear calibration curve used for the estimation of \% release of immobilized fluorescently-labeled hpDNA strands from the surface of Au@PDA NPs as shown in the inset of Figure 2B. Data are recorded from fluorescence measurements of known concentrations of FITC-labeled hpDNA against miR-29b. Data obtained from three independent measurements are presented as mean $\pm \mathrm{SD}$. 


\section{Figure S9}

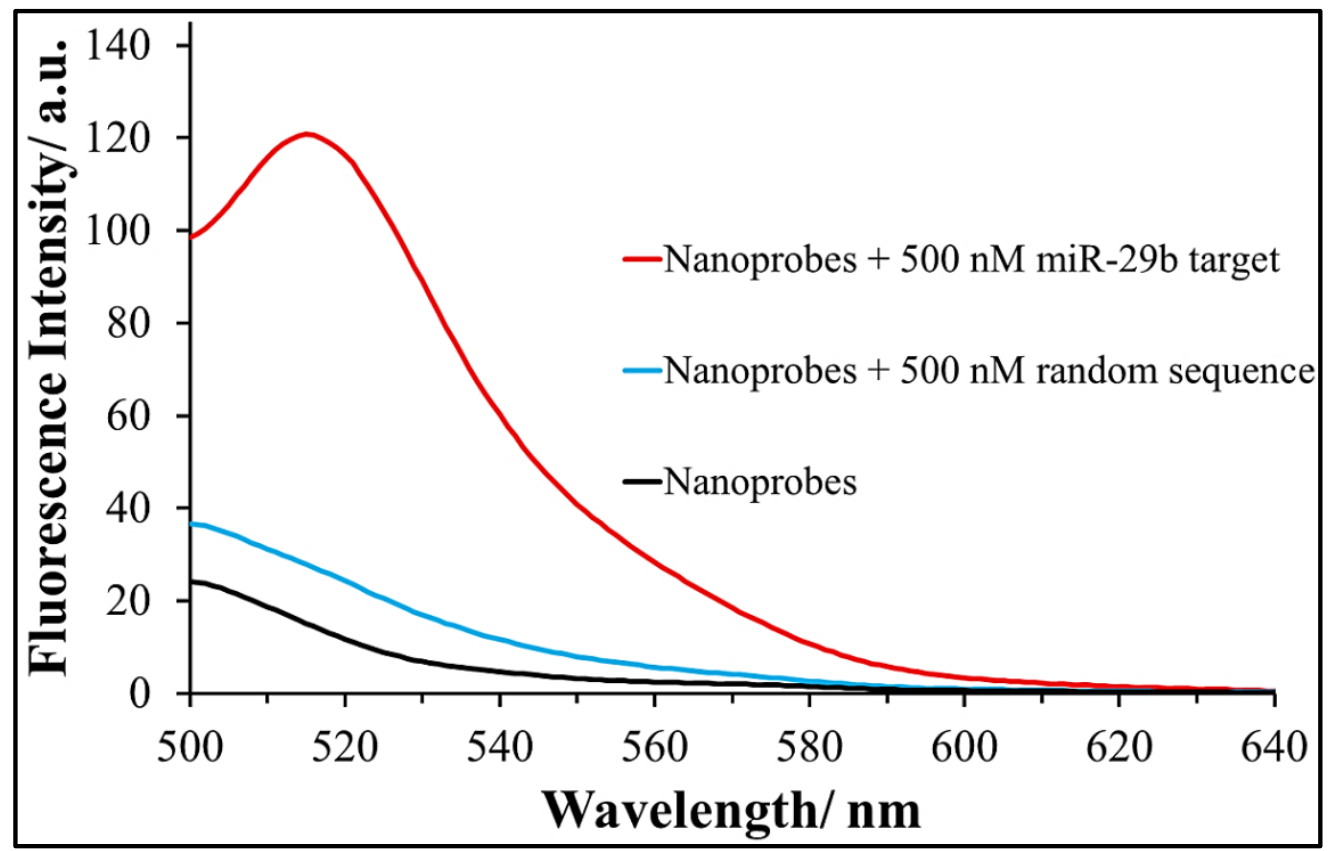

Figure S9. Release assay of Au@PDA-hpDNA nanoprobes in a buffer system. 100 $\mathrm{nM}$ of FITC-labeled hpDNA strands that specifically recognize miR-29b are first immobilized onto Au@PDA NPs to form the nanoprobes against miR-29b. Red trace shows the fluorescence signals of the nanoprobes upon $2 \mathrm{~h}$ of incubation with $500 \mathrm{nM}$ of DNA analog of miR-29b (target). Blue trace shows the fluorescence signals of the nanoprobes upon $2 \mathrm{~h}$ of incubation with $500 \mathrm{nM}$ of random DNA sequence as control. Purple trace shows the fluorescence signal of the nanoprobes itself. These data support that our nanoprobes show reasonable specificity against miR-29b. 


\section{Figure S10}

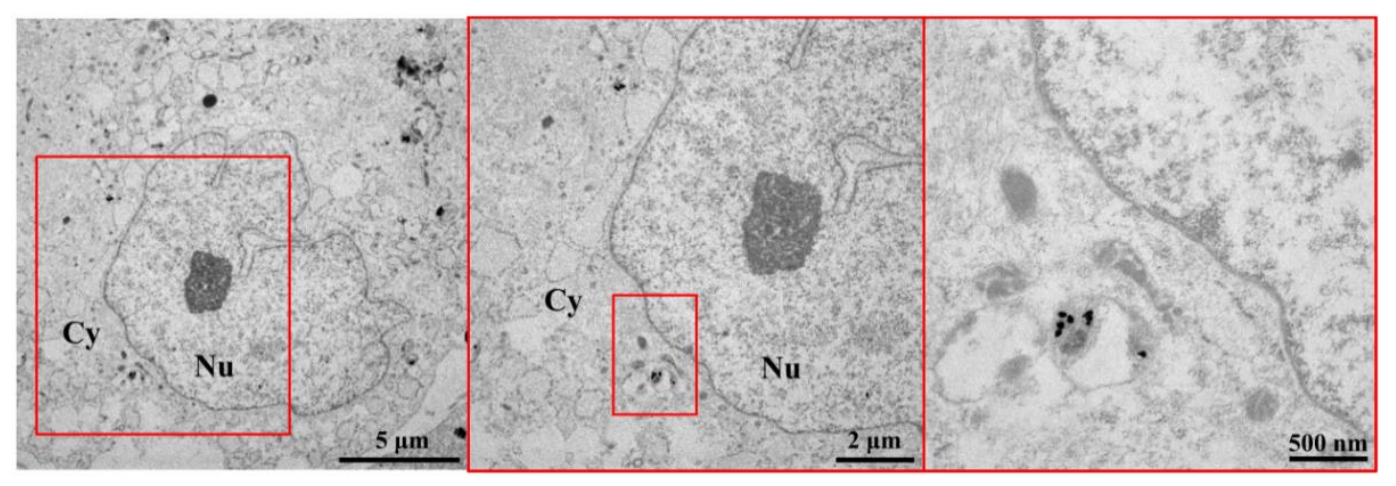

Figure S10. Representative TEM images of hMSCs incubated with Au@ PDA NPs for 24 h. Magnified images show that most uptaken Au@PDA NPs are located in the perinuclear region inside hMSCs. $\mathrm{Cy}=$ cytoplasm, $\mathrm{Nu}=$ nucleus. 


\section{Figure S11}

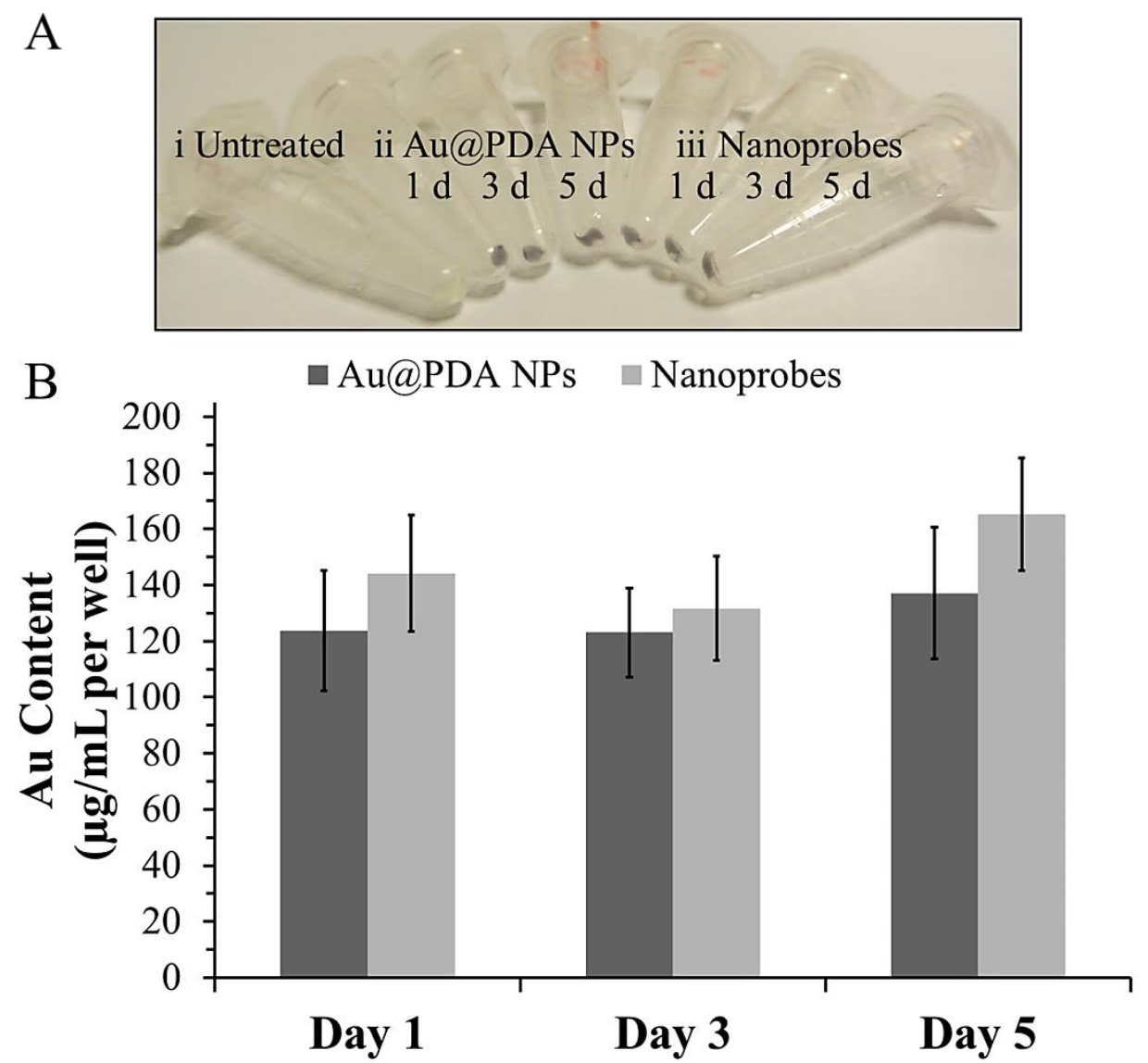

Figure S11. Examination of the Au content inside hMSCs $1 \mathrm{~d}, 3 \mathrm{~d}$, and $5 \mathrm{~d}$ after initial cellular uptake of nanoparticles. hMSCs are treated with nanoparticles for $24 \mathrm{~h}$ before rinsing with PBS. (A) Photographs of cell pellet collected for (i) untreated hMSCs, (ii) Au@PDA NP-treated hMSCs, and (iii) Au@PDA-hpDNA nanoprobe-treated hMSCs. Color of cell pellets for both the Au@PDA NP-treated and nanoprobe-treated hMSCs remain intense as purple in color within the observation time window. (B) ICP-OES measurements show that there is no obvious reduction in the $\mathrm{Au}$ content of cell-associated nanoparticles for both the Au@PDA NP-treated and nanoprobe-treated hMSCs even after $5 \mathrm{~d}$ of incubation, suggesting minimal exocytosis of the nanoparticles. Data obtained from three independent measurements are presented as mean \pm SD. 


\section{Figure S12}

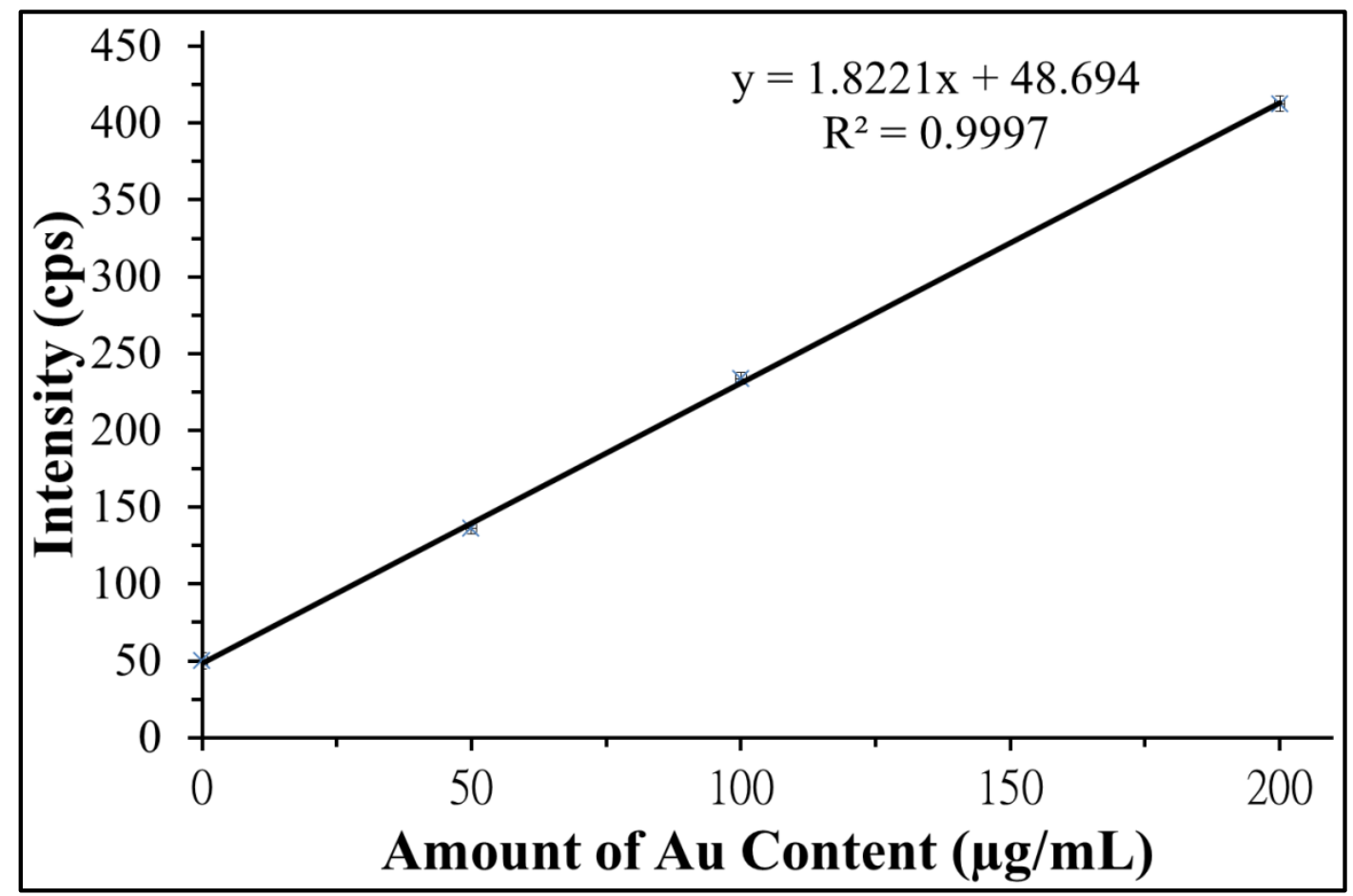

Figure S12. Standard linear calibration curve obtained from the ICP-OES measurements of varying concentration of gold standard solutions. This calibration curve is used to determine the concentration of the stock solution of AuNPs and the $\mathrm{Au}$ content per well as shown in Figure 3C. Data obtained from three independent measurements are presented as mean $\pm \mathrm{SD}$. 


\section{Figure S13}

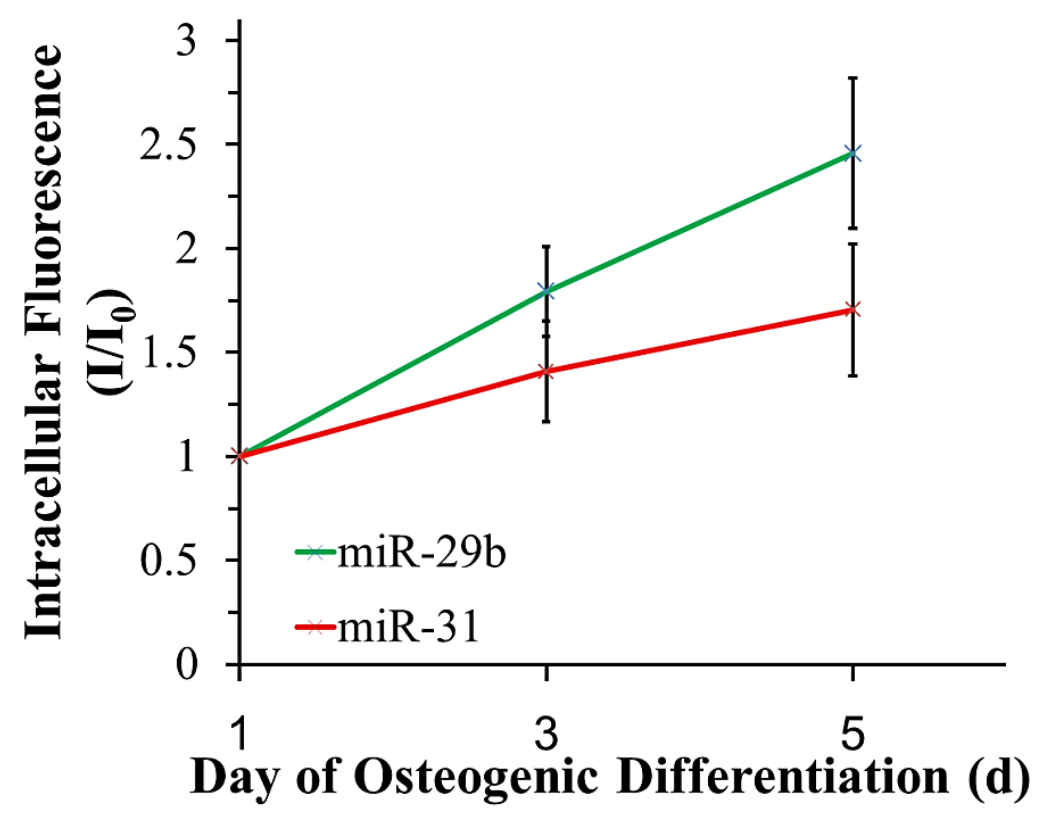

Figure S13. Quantification of intracellular fluorescence of Au@PDA-hpDNA nanoprobe-treated hMSCs upon different days of osteogenic induction. Green trace shows the plot of cell fluorescence associated with the detection of miR-29b as a function of day of osteogenic differentiation. Red trace shows the corresponding plot for the detection of miR-31. A monotonic increasing trend of the intracellular fluorescence along with the osteogenic induction time is observed for both osteogenic marker miRNAs, consistent with our qRT-PCR results of these two miRNAs as shown in Figure 4. Cell fluorescence signals are presented as $I / I_{0}$, where $I_{0}$ represents the mean fluorescence of hMSCs upon $1 \mathrm{~d}$ of osteogenic induction. Data obtained from over 50 cells per sample are presented as mean \pm SD. 


\section{Figure S14}

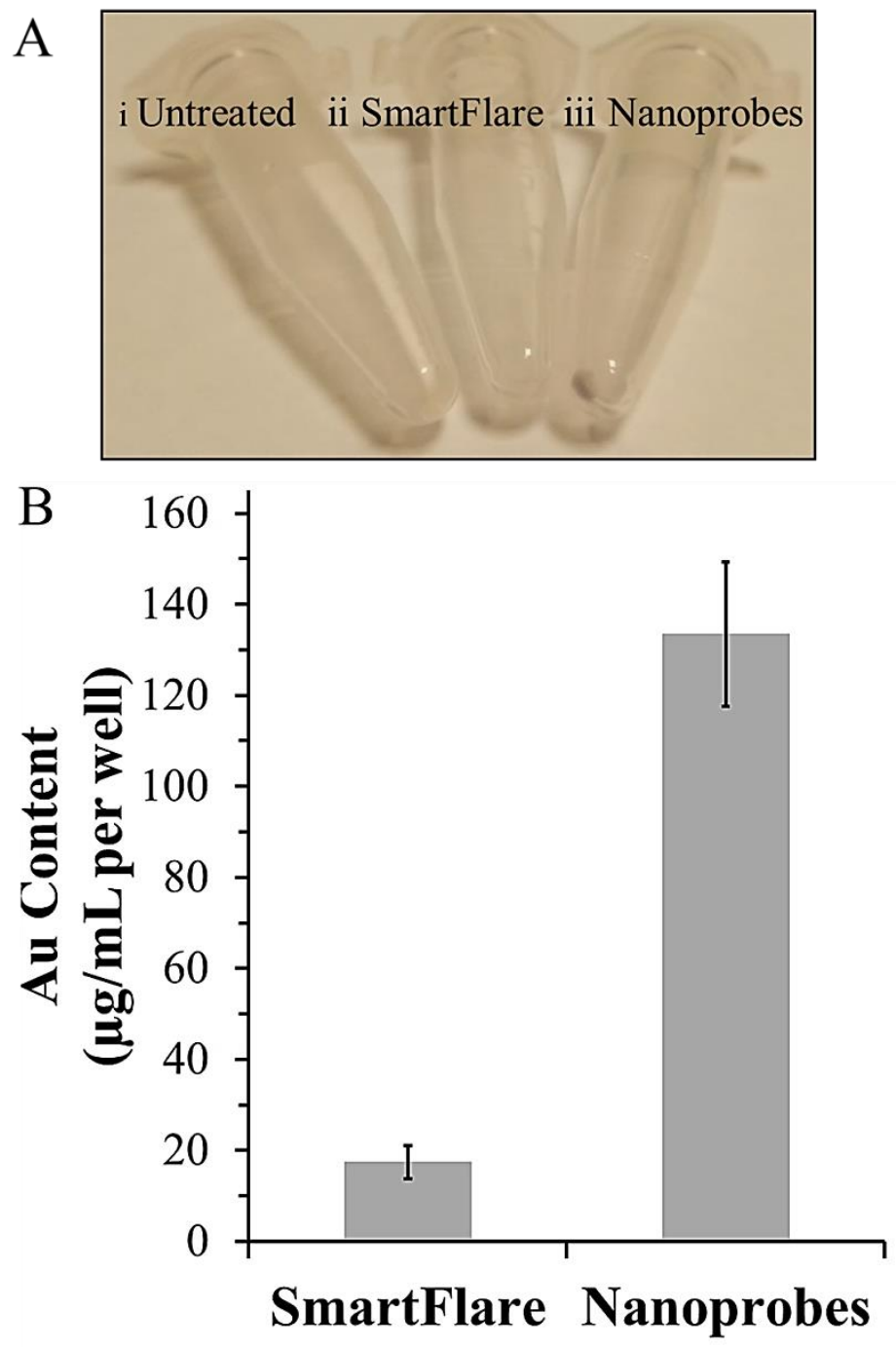

Figure S14. Comparison of cellular uptake property of our Au@PDA-hpDNA nanoprobes (nanoprobes) with the commercial RNA detection probe SmartFlare in hMSCs. hMSCs are treated with nanoparticles for $24 \mathrm{~h}$. Concentrations of the nanoparticles are adjusted to be equivalent at $\sim 0.025 \mathrm{nM}$. (A) Photographs of cell pellets collected for (i) untreated hMSCs, (ii) SmartFlare-treated hMSCs, and (iii) nanoprobe-treated hMSCs. Intense color is observed only for the nanoprobe-treated cell pellet but not the SmartFlare-treated cell pellet. (B) ICP-OES measurements show that the $\mathrm{Au}$ content in the nanoprobe-treated cell samples is $\sim 7$-fold higher than that in the SmartFlare-treated cell samples, indicating that our nanoprobes are more efficiently internalized by hMSCs. Data obtained from three independent measurements are presented as mean \pm SD. 


\section{Figure S15}

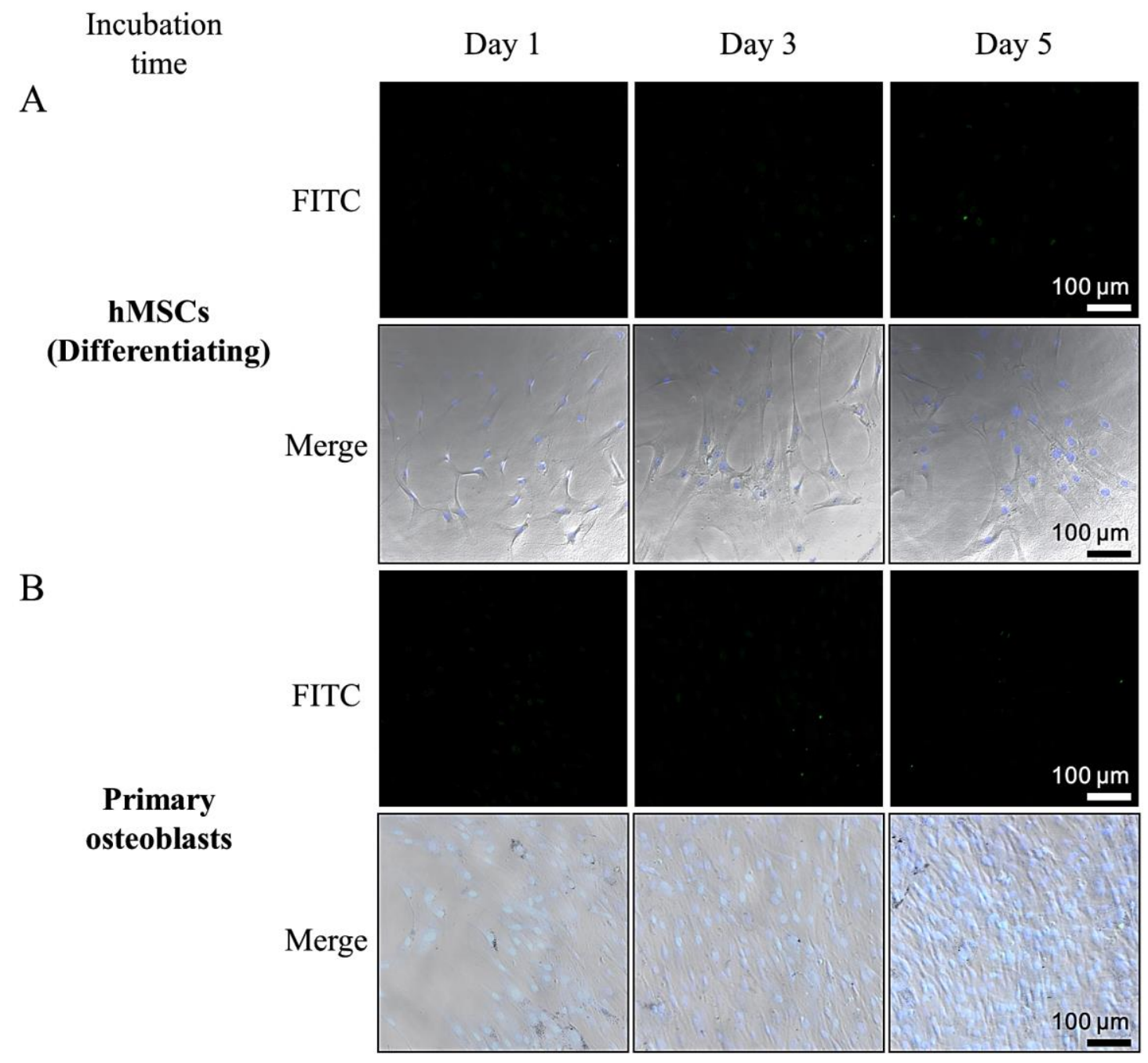

Figure S15. Intracellular specificity and stability of Au@PDA-hpDNA nanoprobes. $500 \mathrm{nM}$ of the FITC-labeled scrambled DNA strands are immobilized onto Au@PDA NPs and then incubated with (A) hMSCs and (B) primary osteoblasts for $24 \mathrm{~h}$. Cells are imaged at the selected time points after incubation. Results show negligible fluorescence responses in both the differentiating hMSCs and osteoblasts, indicating that our nanoprobes are specific and resistant to nuclease degradation for at least 5 days of culture. Nuclei were counter-stained with Hoechst 33342. Scale bar is $100 \mu \mathrm{m}$. DAPI = 4,6-diamidino-2-phenylindole. 


\section{Scheme S1}
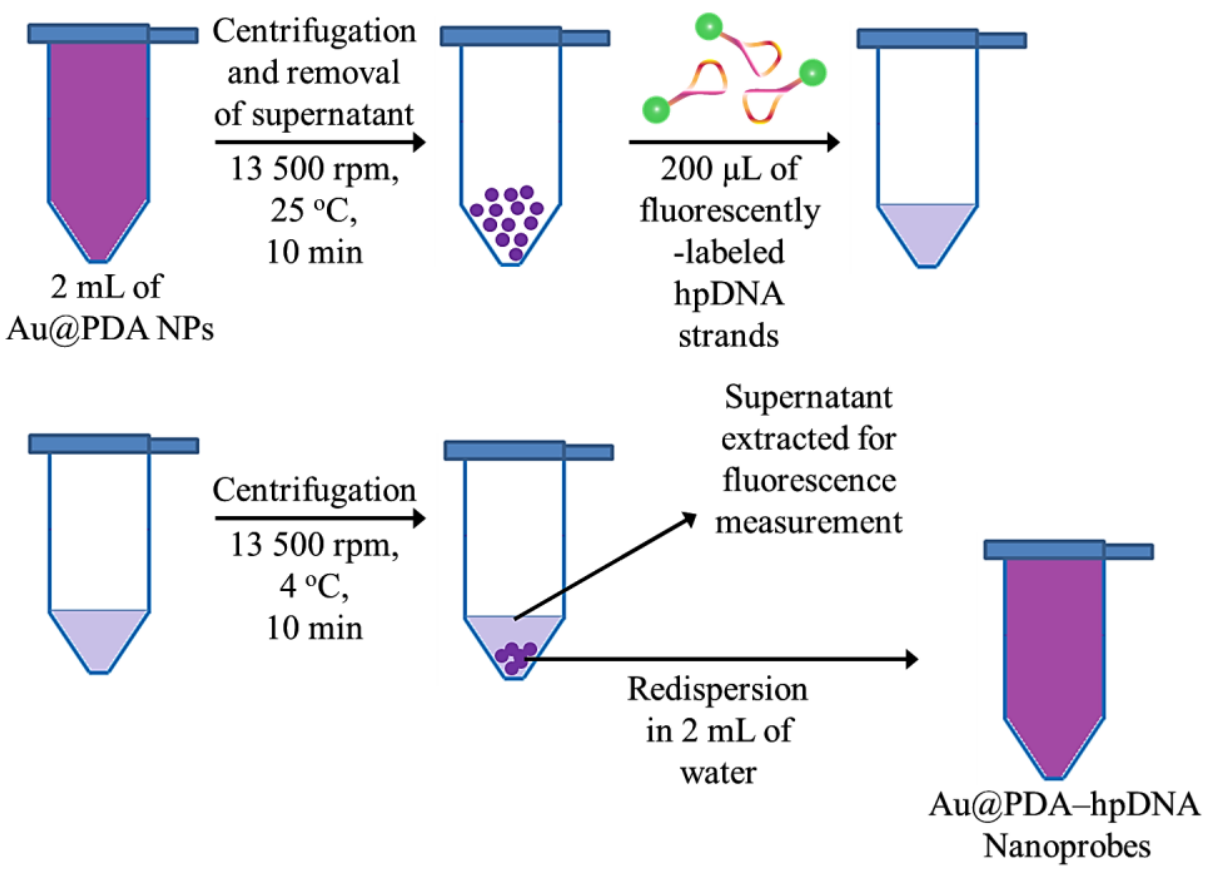

Scheme S1. Monitoring of the immobilization of fluorescently-labeled hpDNA strands onto the surface of Au@PDA NPs. All nanoprobes used in this study are prepared in the same manner as described. 


\section{REFERENCES}

(1) Khlebtsov, N. G. Anal. Chem. 2008, 80, 6620. 